\title{
A Case Study of Computer Analysis of the Arthritic User in Rehabilitation Engineering
}

\author{
G. Jeffries, D.K. Wright, N. Rogers and K. Leibrandt. \\ Dept. of Design, Brunel University, London, U.K.
}

\section{Introduction}

In this work we have combine the use of anthropometric data and mechanical analysis software to model a complete system in the form of a user combined with a product. Analysis of the system is then possible, to find the forces, moments, accelerations, and any other dynamic information. Applying existing maximum force and moment criteria, within an iterative process, we are able to evaluate different configurations of a product. This method of analysis can be used either to examine the quality of the interface on an existing product, or during the concept stage in the design of a new product.

\section{Case Study}

The subject of this study is a lady in her late seventies, with Rheumatoid Arthritis in her wrists, and also Osteo-arthritis in her back. An android (body segment model) was built to represent the user, corresponding to body measurements. A model of a cleaner was generated using a solid modeller, with the correct centres of gravity, mass distributions and moments of inertia. The two models were then combined by a connector at the hand-handle interface. The system was analysed with motion data taken from video images, driving the cleaner through space. The analysis data is then used to drive the Android in the form of an inverse kinematics analysis. Results are generated for the velocity, accelleration, and forces generated in the body segments and joints. From these it was possible to evaluate the effect of different configurations of a product, for example the texture, angle, size and shape of the cleaner handle. For example, in this user it was seen that the force generated in the shoulder could be reduced by increasing the angle of the handle to the vertical. Work is now being carried out to produce prototypes, as the next stage of the design process. These will be evaluated by a user group, and the results compared with that of the analysis.

\section{Conclusions}

From the data obtained by this method of analysis, and applying existing anthropometric and ergonomic principles, products can be evaluated with comparison to existing studies. This makes feasible the study of complex 3D systems, and also provides a valuable evaluation tool in the design process. Further development of this tool will incorporate joint range of motion and time dependent criteria such as fatigue. 